\title{
Magneto-optic spectroscopy with linearly polarized modulated light: Theory and experiment
}

\author{
G. Bevilacqua* \\ Department of Information Engineering and Mathematical Science, University of Siena, Via Roma 56, 53100 Siena, Italy \\ E. Breschi ${ }^{\dagger}$ \\ Department of Physics, University of Fribourg, Chemin du Musée 3, 1700 Fribourg, Switzerland
}

(Received 11 April 2014; published 18 June 2014)

\begin{abstract}
We investigate the polarization modulation between two linear orthogonal states of the laser beam that synchronously pumps time-dependent atomic alignment in caesium atoms exposed to a static magnetic field. Because of the atomic alignment symmetry two independent groups of resonances can be distinguished in the transmitted light: when modulation frequency matches either the Larmor frequency or its second harmonics, $\omega_{L}$ and $2 \omega_{L}$, respectively. We report on our experiments, and discuss a model that perfectly reproduces the observed spectra. We have observed that the amplitudes and linewidths of resonances at $\omega_{L}$ and $2 \omega_{L}$ show magnetic-field direction dependence. This peculiar behavior makes our approach interesting for future application to atomic magnetometry, in view of a dead-zone free high-sensitivity magnetometer.
\end{abstract}

DOI: 10.1103/PhysRevA.89.062507 PACS number(s): 32.30.Dx, 32.60.+i, 32.70.Jz, 33.40.+f

\section{INTRODUCTION}

Magneto-optical resonances are observed in an atomic ensemble of spin polarized atoms in the presence of an external magnetic field, B. The Larmor precession induced by $\mathbf{B}$ modifies the absorption and dispersion properties of the atomic ensemble, and the magneto-optical resonances can be recorded probing the properties (intensity or polarization) of the transmitted light beam. A comprehensive review of the magneto-optical resonances has been done in Ref. [1]. The combination of simultaneous pumping and probing can be exploited in a wide range of applications such as metrology and quantum information. In particular it has been used to measure magnetic fields since the 1960s [2]. During the 1990s, thanks to the development of compact and reliable diode lasers, atomic magnetometry had a renewed interest. Replacing spectral lamps with lasers allowed studying new approaches based on the modulation of light parameters that was in the past limited to amplitude modulation [3]. Relevant contributions in frequency- and amplitude-modulation spectroscopy comes from different groups [4-7]. In recent years atomic magnetometers [8] have become valuable tools not only in general physics [9], but also in exotic applications like detection of magnetic particles [10], and ultra-low-field NMR [11,12], etc.

Among the various configurations to excite magnetooptical resonances in atoms or molecules based optical pumping with resonant light [1], we focus our attention on resonant polarization modulation technique which was poorly studied in the past.

In Ref. [13], it is shown that modulating the light polarization between linear and circular polarization states simultaneously excites at the same frequency alignment and orientation atomic moments, and it allows the elimination of both the dead-zone and heading errors. This approach however suffers of sensitivity losses for given directions of $\mathbf{B}$.

\footnotetext{
*giuseppe.bevilacqua@unisi.it

†evelina.breschi@unifr.ch
}

Last year polarization modulation between two right- and left-circular polarizations has been largely studied: a simplified theoretical model is reported in Ref. [14], the full characterization of the magneto-optical spectrum in Refs. $[15,16]$, and its application to atomic magnetometry in Ref. [17]. Symmetric right- and left-circular polarization modulation is particularly efficient because the pumping light is modulated synchronously with the spin evolution dynamics induced by B. This is the concept behind the push-pull optical pumping originally proposed to increase the contrast of magnetic insensitive resonance (atomic clock applications) [18]. In this paper we address the effect on the atomic ensemble of a symmetric modulation of the laser polarization between linear orthogonal states.

The interaction geometry we discuss from the theoretical and experimental point of view is shown in Fig. 1. The laser beam propagates along $\mathbf{k}$, and the polarization $\boldsymbol{\epsilon}$ is modulated at frequency $\Omega$ between two orthogonal linear polarization states: the $\epsilon_{x}$ and $\epsilon_{y}$ components of the polarization vector, respectively. The magnetic field, whose strength is kept constant, can be applied in an arbitrary direction.

The paper is organized as follows. In Sec. II we describe the theoretical model developed for the data interpretation leaving cumbersome calculation details to the Appendix. The main characteristic of the experimental apparatus and data treatment are given in Sec. III. The full spectra of resonances detected with phase-sensitive detections and their dependence on the magnetic-field direction are reported in Secs. IV and V, respectively.

\section{THEORETICAL MODEL}

To properly model the system, the full optical Bloch equations for the transition $F_{g} \rightarrow F_{e}$, have to be solved in the presence of a magnetic field. In general this is a difficult task, and can be pursued only by numerical methods, often obfuscating the physical insight. Hence introducing relevant approximations will both simplify the calculations and make transparent the physical mechanisms involved. 


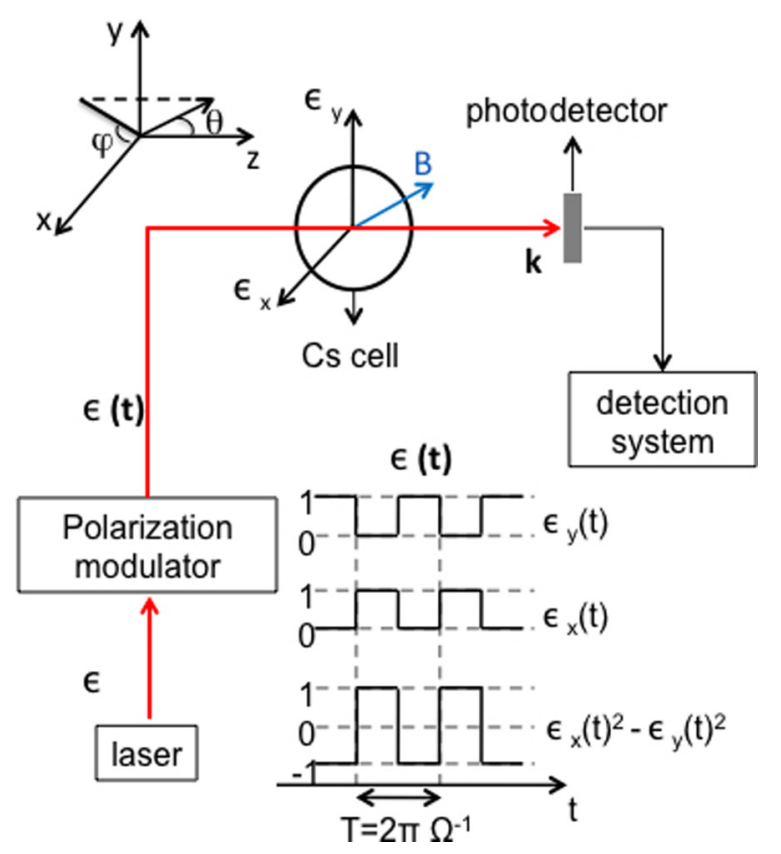

FIG. 1. (Color online) The interaction geometry. The laser beam propagating along the $z$ axis passes through the caesium cell kept at room temperature. The transmitted signal is recorded and analyzed in standard way. A magnetic field whose strength is constant, and the direction can be varied, is applied to the atoms, The Larmor frequency $\omega_{L}$ is typically about $2 \pi \times 2 \mathrm{kHz}$. The beam polarization is switched between two orthogonal states via a polarization modulator: the horizontal $\left(\epsilon_{x}\right)$ and the vertical $\left(\epsilon_{y}\right)$ linear polarization, at a rate $\Omega$ which is swept across the resonances. In the lower part is sketched the time dependence of the polarization vector $\epsilon$ components.

The first approximation is dictated by the low laser power (about $2 \mu \mathrm{W}$ for our experimental condition), which suggests to treat the atom-laser interaction in a perturbative way. Second, we observe that the interaction has two characteristic time scales that differ by several orders of magnitude: (i) the laser optical pumping cycle in the $\mu$ s range, and (ii) the ground-state dynamics determined by $\omega_{L}$ and $\Omega$ in the ms range. Practically the optical pumping is instantaneous on the ms time scale allowing us to decouple the effect of the laser pumping and of the magnetic field. As a consequence the effect of laser light can be taken into account by means of pumping rates on the ground Zeeman multiplet, neglecting the excited Zeeman multiplet, allowing us to substantially simplify the initial problem [19].

The optical properties of the atomic ensemble are described by the linear absorption coefficient $\kappa$ which can be opportunely used to compare theoretical and experimental results. The $\kappa$ is connected to the ground-state multipoles [20,21], and reads

$$
\kappa \propto E_{0}^{2} \sum_{k, q}(-1)^{k+q}\left(m_{q}^{k}\right)^{*} \mathcal{A}_{k} \mathbb{E}_{-q}^{k},
$$

where the ground state and laser field multipole moments $m_{q}^{k}$ (see the Appendix for the definition) and $\mathbb{E}_{-q}^{k}$ are introduced. These latter are defined in terms of the laser polarization vector as

$$
\begin{aligned}
\mathbb{E}_{Q}^{K}= & (-1)^{K+Q} \sqrt{2 K+1} \sum_{q, q^{\prime}=-1}^{1}\left(\begin{array}{ccc}
1 & 1 & K \\
q & q^{\prime} & Q
\end{array}\right) \\
& \times\left(\epsilon^{*}\right)_{-q} \epsilon_{-q^{\prime}} .
\end{aligned}
$$

The constants $\mathcal{A}_{k}$ are not important in the following and will be discarded. Note that $\kappa$ is time dependent because both $\mathbb{E}_{-q}^{k}$ and $m_{q}^{k}$ are time dependent.

With our axis convention (Fig. 1) $\epsilon_{z}=0$ while $\epsilon_{x}$ and $\epsilon_{y}$ are real quantities having the time behavior sketched in the right part of Fig. 1, and by using definition (2), one can see that the only nonzero laser field multipoles are $\mathbb{E}_{0}^{0}=-1 / \sqrt{3}$, $\mathbb{E}_{0}^{2}=-1 / \sqrt{6}$, and $\mathbb{E}_{ \pm 2}^{2}=\left(\epsilon_{x}^{2}-\epsilon_{y}^{2}\right) / 2 \equiv f(t) / 2$, and then $\kappa$ becomes

$$
\kappa=-\frac{1}{\sqrt{6}} m_{0}^{2}(t)+\frac{f(t)}{2}\left[m_{2}^{2}(t)+m_{-2}^{2}(t)\right],
$$

where we have dropped the total population $\left(m_{0}^{0}\right)$ contribution, and the proportionality constant $\mathcal{A}_{2}$. The function $f(t)$ represents a symmetric square wave signal oscillating between -1 and +1 with angular frequency $\Omega$. Its Fourier series is given by $f(t) \equiv \sum_{n=-\infty}^{+\infty} f_{n} e^{i n \Omega t}=\frac{4}{\pi} \sum_{n>0, \text { odd }} \frac{1}{n} \sin (n \Omega t)$. Explicitly $f_{2 n+1}=-(2 i / \pi) /(2 n+1), f_{2 n}=0$, and $f_{-n}=$ $f_{n}^{*}=-f_{n}$.

In the presence of a magnetic field and of relaxation mechanisms, the $m_{q}^{k}$ satisfy the following equations [20]:

$$
\begin{aligned}
\dot{m}_{q}^{k}= & \left(i \omega_{0} q-\gamma_{q}^{(k)}\right) m_{q}^{k}+i \frac{\omega_{-}}{2} c_{k, q-1}^{+} m_{q-1}^{k} \\
& +i \frac{\omega_{+}}{2} c_{k, q+1}^{-} m_{q+1}^{k}+S_{q}^{k},
\end{aligned}
$$

where $\quad k=0, \ldots, 2 F_{g}, \quad q=-k, \ldots, k, \quad$ and $\quad c_{k, q}^{ \pm} \equiv$ $\sqrt{k(k+1)-q(q \pm 1)} ; \omega_{0}=g_{F_{g}} \mu_{B} B_{z} \equiv g_{F_{g}} \mu_{B} B \cos \theta$ and $\omega_{ \pm}=g_{F_{g}} \mu_{B}\left(B_{x} \pm i B_{y}\right) \equiv g_{F_{g}} \mu_{B} B \sin \theta e^{ \pm i \phi}$ are the longitudinal and transverse Larmor frequency components, respectively. The pumping rates $S_{q}^{k}(t)$,

$$
\left(\frac{d m_{q}^{k}}{d t}\right)_{\text {laser }} \equiv S_{q}^{k}(t) \propto(-1)^{q} \mathbb{E}_{-q}^{k},
$$

act as source terms in the evolution equations of the multipole moments. The full derivation of the previous equation is given in the Appendix. We stress that the rates $S_{q}^{k}(t)$ depend on time because the polarization vector is modulated in the $\mathrm{kHz}$ range. Essentially we are assuming that Eq. (5) fully describes the optical pumping in the ground-state Zeeman multiplet.

The relaxations rates $\gamma_{q}^{(k)}$ as usual describe the effect of relaxation mechanisms on the ground state. Typically for atomic vapor in a glass cell the main relaxation mechanisms are due to collisions between alkali-metal atoms and buffer gas or cell walls (coated or uncoated) [22]. As dictated by (3), to describe our experiments we need to solve Eq. (4) for $k=2$. Simplifying the notation $\gamma_{q}^{(2)} \equiv \gamma_{q}$, we neglect the associated frequency shifts, thus, the $\gamma_{q}$ are real and positive and they satisfy the relation $\gamma_{q}=\gamma_{-q}$ to ensure the density matrix Hermiticity.

For convenience we introduce the vectors $\mathbf{x}=$ $\left(m_{-2}^{2}, m_{-1}^{2}, m_{0}^{2}, m_{1}^{2}, m_{2}^{2}\right)$ for the $k=2$ multipole moments and 
$\mathbf{u}=(f(t), 0,-2 / \sqrt{6}, 0, f(t))$ for the pumping rates. Then equations (4) can be cast as

$$
\dot{\mathbf{x}}(t)=A \mathbf{x}(t)+\mathbf{u}(t)
$$

leading to the solution

$$
\mathbf{x}(t)=e^{A t} \mathbf{x}(0)+e^{A t} \int_{0}^{t} e^{-A t^{\prime}} \mathbf{u}\left(t^{\prime}\right) d t^{\prime} .
$$

The matrix $A$ represents the dynamics of the quadrupole moment in a magnetic field in the presence of relaxation mechanisms; its explicit form can be derived from Eqs. (4). By direct inspection of its characteristic polynomial one can see that the eigenvalues of $A, \lambda_{k}(k=1, \ldots, 5)$, are real or complex conjugate couples. The $\lambda_{k}$ have a negative real part, and thus the initial conditions in the right-hand side of Eq. (7) decay exponentially, and in the long-time limit $\left(t \gg\left|1 / \operatorname{Re}\left(\lambda_{k}\right)\right|\right)$ can be neglected.

To efficiently evaluate the remaining integral we introduce the diagonal form of $A=U D U^{-1}$, and we use the explicit expression of $\mathbf{u}$ deriving, then (in the long time limit), the complete solution

$$
\begin{aligned}
x_{i}= & \frac{2}{\sqrt{6}} \sum_{k=1}^{5} \frac{U_{i, k} U_{k, 3}^{-1}}{\lambda_{k}} \\
& +\sum_{\substack{n \neq 0 \\
k=1, \ldots, 5}} f_{n} \frac{U_{i, k}\left(U_{k, 1}^{-1}+U_{k, 5}^{-1}\right)}{i n \Omega-\lambda_{k}} e^{i n \Omega t} .
\end{aligned}
$$

After some tedious but straightforward algebra the absorption coefficient can be rewritten as

$$
\kappa=Z_{0}+\sum_{q>0}\left[Z_{+, q} \cos (q \Omega t)+Z_{-, q} \sin (q \Omega t)\right] .
$$

The $Z_{0}$ is the amplitude of the de signal, while $Z_{+, q}$ and $Z_{-, q}$ represent the in-phase and quadrature parts of the demodulated signal at the $q$ th harmonic of the $\Omega$. Explicit and cumbersome expressions for the $Z_{ \pm, q}$ coefficients can be obtained using Eqs. (8), which we prefer to not report in the general case, because they can be noticeably simplified looking at the characteristic values of the model parameters used in the experiment.

All experiments are carried out with the atomic ensemble contained in a paraflint wall coated cell, thus the relaxation rates of multipole moment $m_{q}^{k}, \gamma_{q}$, are all $\leqslant 10 \mathrm{~Hz}$ [22]. On the other hand $\omega_{L}$ is in the $\mathrm{kHz}$ range, i.e., several orders of magnitude larger than the relaxation rates. It is then convenient to define a perturbative parameter $\eta$ as the ratio between the longitudinal (or population) relaxation rate, $\gamma_{0}$, which is the smaller relaxation rate, and $\omega_{L}$, i.e., $\eta=\gamma_{0} / \omega_{L} \ll 1$. This allows us to find a compact expression for both the eigenvalues $\lambda_{k}$ and the matrix $U$. In fact rewriting the matrix $A$ as $A=\omega_{L}\left(i A_{0}-\eta G\right)$ one finds that the matrix $A_{0}$ is diagonalized by a rotation that brings the quantization axis $z$ along to the magnetic field. Thus the diagonal form $A_{0, D}=$ $W^{-1} A_{0} W$ represents the Zeeman effect and its eigenvalues are $-2,-1,0,1,2$. The rotation is expressed by the Wigner matrix $W$ [23]. The matrix $G$ is diagonal and represents the relaxation. Its diagonal elements are $\gamma_{q} / \gamma_{0}$. By factoring the eigenvectors matrix as $U=W V$ we find

$$
D=\omega_{L} V^{-1}\left[i A_{0, D}-\eta W^{-1} G W\right] V
$$

and $W^{-1} G W$ is no longer diagonal. We assumed that $V=$ $\mathbb{1}+\eta V_{1}+\eta^{2} V_{2}+\cdots$ and $D=\omega_{L}\left(i A_{0, D}+\eta D_{1}+\eta^{2} D_{2}+\right.$ $\cdots)$ and proceed by applying the usual perturbation theory [23]: numerically we found that the first-order eigenvalues and the zero-order eigenvectors describe the experimental results. We can then approximate $V \approx \mathbb{1}, U \approx W$, and $D \approx$ $\omega_{L}\left(i A_{0, D}+\eta D_{1}\right)$. Finally by writing $\lambda_{k}=i \alpha_{k}-\Gamma_{k}$ the firstorder eigenvalues are $\alpha_{1}=2 \omega_{L}, \alpha_{2}=\omega_{L}, \alpha_{3}=0$, and

$$
\begin{aligned}
\frac{\Gamma_{1}}{\gamma_{0}}= & 1+\frac{5}{16} g_{1}+\frac{35}{64} g_{2}+\frac{7 g_{2}-4 g_{1}}{16} \cos (2 \theta) \\
& +\frac{g_{2}-4 g_{1}}{64} \cos (4 \theta), \\
\frac{\Gamma_{2}}{\gamma_{0}}= & 1+\frac{1}{2} g_{1}+\frac{5}{16} g_{2} \\
& -\frac{g_{2}-g_{1}}{4} \cos (2 \theta)-\frac{g_{2}-4 g_{1}}{16} \cos (4 \theta), \\
\frac{\Gamma_{3}}{\gamma_{0}}= & 1+\frac{9}{32} g_{2}+\frac{3}{8} g_{1}-\frac{3 g_{2}}{8} \cos (2 \theta) \\
& -\frac{12 g_{1}-3 g_{2}}{32} \cos (4 \theta),
\end{aligned}
$$

where $g_{i}=\gamma_{i} / \gamma_{0}-1$. Remind that $\lambda_{5}=\lambda_{1}^{*}$ and $\lambda_{4}=\lambda_{2}^{*}$.

We stress that in the simplified case of isotropic relaxation, i.e., $\gamma_{2}=\gamma_{1}=\gamma_{0}\left(g_{i} \equiv 0\right)$, often solved in literature [24], $G$ is the identity matrix, and then $W^{-1} G W$ remains diagonal. As a consequence the eigenvalues are exactly $\lambda_{1}=2 i \omega_{L}-\gamma_{0}$, $\lambda_{2}=i \omega_{L}-\gamma_{0}, \lambda_{3}=-\gamma_{0}$. Essentially imposing $\gamma_{i}$ equal corresponds to neglect the linewidth dependence in the magnetic-field direction.

Using the explicit form of $U$ and $\lambda_{k}$ and the Lorentzian $\mathcal{L}$ and dispersive $\mathcal{D}$ profiles,

$$
\begin{aligned}
\mathcal{L}_{ \pm 1, p}-i \mathcal{D}_{ \pm 1, p} & =\frac{1}{i\left(\Omega \mp \omega_{L} / p\right)+\Gamma_{2} / p} \\
\mathcal{L}_{ \pm 2, p}-i \mathcal{D}_{ \pm 2, p} & =\frac{1}{i\left(\Omega \mp 2 \omega_{L} / p\right)+\Gamma_{1} / p} \\
\mathcal{L}_{0, p}-i \mathcal{D}_{0, p} & =\frac{1}{i \Omega+\Gamma_{3} / p}
\end{aligned}
$$

we find $Z_{+, q}$ and $Z_{-, q}$ describing our experiment.

In the following we present the results for odd and even detection harmonics, i.e., the harmonics of the modulation frequency used to demodulate the signal; see Eq. (9).

Odd detection harmonics. Defining the coefficients

$$
\begin{aligned}
M_{1}^{(R)} & =\frac{\sqrt{6}}{8} \cos 2 \phi \sin ^{2} \theta\left(1+\cos ^{2} \theta\right), \\
M_{1}^{(I)} & =-\frac{\sqrt{6}}{4} \sin 2 \phi \sin ^{2} \theta \cos \theta, \\
M_{2}^{(R)} & =-\frac{\sqrt{6}}{2} \cos 2 \phi \sin ^{2} \theta \cos ^{2} \theta, \\
M_{2}^{(I)} & =-2 M_{1}^{(I)} \\
M_{3} & =\frac{\sqrt{6}}{4} \cos 2 \phi \sin ^{2} \theta\left(3 \cos ^{2} \theta-1\right),
\end{aligned}
$$


the in-phase and quadrature parts of the demodulated signal reads

$$
\begin{aligned}
\frac{\sqrt{6} \pi q^{2}}{4} Z_{+, q}^{\text {odd }}= & {\left[M_{1}^{(I)} \mathcal{L}_{-2, q}+M_{1}^{(R)} \mathcal{D}_{-2, q}\right] } \\
& +\left[-M_{1}^{(I)} \mathcal{L}_{2, q}+M_{1}^{(R)} \mathcal{D}_{2, q}\right] \\
& +\left[M_{2}^{(I)} \mathcal{L}_{-1, q}+M_{2}^{(R)} \mathcal{D}_{-1, q}\right] \\
& +\left[-M_{2}^{(I)} \mathcal{L}_{1, q}+M_{2}^{(R)} \mathcal{D}_{1, q}\right] \\
& +M_{3} \mathcal{D}_{0, q}, \\
\frac{\sqrt{6} \pi q^{2}}{4} Z_{-, q}^{\text {odd }}= & -\left\{\left[M_{1}^{(R)} \mathcal{L}_{2, q}+M_{1}^{(I)} \mathcal{D}_{2, q}\right]\right. \\
& +\left[M_{1}^{(R)} \mathcal{L}_{-2, q}-M_{1}^{(I)} \mathcal{D}_{-2, q}\right] \\
& +\left[M_{2}^{(R)} \mathcal{L}_{1, q}+M_{2}^{(I)} \mathcal{D}_{1, q}\right] \\
& +\left[M_{2}^{(R)} \mathcal{L}_{-1, q}-M_{2}^{(I)} \mathcal{D}_{-1, q}\right] \\
& \left.+M_{3} \mathcal{L}_{0, q}\right\}+\frac{\sqrt{6} q}{2} \text { offset. }
\end{aligned}
$$

These expressions clearly show that the spectrum (as a function $\Omega$ ) is symmetric around $\Omega=0$, and is composed of exactly five resonances occurring at $\Omega= \pm 2 \omega_{L} / q, \Omega= \pm \omega_{L} / q$, and $\Omega=0$, respectively. Each resonance, apart that at $\Omega=0$, is neither Lorentzian nor dispersive but a mixing of both types. Finally in the quadrature part of the signal $\left(Z_{-, q}^{\text {odd }}\right)$ one can observe a $\Omega$-independent background.

Even detection harmonics. The case of even harmonics is more structured. We obtain

$$
\begin{aligned}
Z_{+, q}^{\text {even }} \propto & -\sum_{n>0, \text { odd }} \frac{1}{n\left(q^{2}-n^{2}\right)}\left\{C_{1}\left[\mathcal{L}_{-2, n}+\mathcal{L}_{2, n}\right]\right. \\
& \left.+C_{2}\left[\mathcal{L}_{-1, n}+\mathcal{L}_{1, n}\right]+C_{3} \mathcal{L}_{0, n}\right\} \\
Z_{-, q}^{\text {even }} \propto & -\sum_{n>0, \text { odd }} \frac{q}{n^{2}\left(q^{2}-n^{2}\right)}\left\{C_{1}\left[\mathcal{D}_{-2, n}+\mathcal{D}_{2, n}\right]\right. \\
& \left.+C_{2}\left[\mathcal{D}_{-1, n}+\mathcal{D}_{1, n}\right]-C_{3} \mathcal{D}_{0, n}\right\}
\end{aligned}
$$

The spectra are symmetric and composed of a superposition of two series of resonances at $\pm 2 \omega_{L}, \pm 2 \omega_{L} / 3, \pm 2 \omega_{L} / 5$, etc., and at $\pm \omega_{L}, \pm \omega_{L} / 3, \pm \omega_{L} / 5$, etc. This is due to the fact that the $n$th odd harmonics of $f(t)$ goes in resonance at $2 \omega_{L} / n$ (or $\left.\omega_{L} / n\right)$. The lack of even harmonics in $f(t)$ prevents a similar structure in the $Z_{ \pm, q}^{\text {odd }}$. Equations (17) also show that the line shape of resonances is purely absorptive and purely dispersive. Again the explicit form of $C_{k}$ coefficients is

$$
\begin{aligned}
& C_{1}=-\frac{1}{8}(1-\cos 4 \phi) \sin ^{4} \theta+\frac{1}{4}\left(1+\cos ^{2} \theta\right)^{2}, \\
& C_{2}=\frac{1}{2}(1-\cos 4 \phi) \sin ^{4} \theta+\frac{1}{4} \sin ^{2}(2 \theta), \\
& C_{3}=\frac{3}{4}(1+\cos 4 \phi) \sin ^{4} \theta .
\end{aligned}
$$

\section{EXPERIMENTAL APPARATUS AND DATA ANALYSIS}

The experimental apparatus has been described in detail in several recent papers; see [16] and references therein. Here for the sake of completeness we overview the fundamental features.
The polarization of a distributed feedback laser whose optical frequency is stabilized on the $F_{g}=4 \rightarrow F_{e}=3$ transition of the cesium $D_{1}$ line $\left(6 S_{1 / 2} \rightarrow 6 P_{1 / 2}\right)$, is modulated via an electro-optical modulator driven with a symmetric square wave ( $50 \%$ duty cycle). The polarization of the beam is then essentially switched between horizontal $\left(\epsilon_{x}\right)$ and vertical $\left(\epsilon_{y}\right)$ linear polarizations. The degree of linear polarization is measured and approaches $98 \%$ for both states. We point out that spurious circular polarization components would add resonances due to the atomic orientation as studied in Ref. [16]. The laser power is about $P_{L} \approx 2 \mu \mathrm{W}$ and the spot diameter is $4 \mathrm{~mm}$ for all experiments, so that $\Omega_{R} / \Gamma \approx 0.13$ thus fulfilling the theory validity condition (see the Appendix). We remark that for each measurement $P_{L}$ is independently monitored in order to determine the value within a few percent.

The cesium vapor is contained into a paraflint coated glass-blow spherical cell with a diameter of $30 \mathrm{~mm}$ [25]. The cell is at room temperature (about $20^{\circ} \mathrm{C}$ ). The laser beam diameter is noticeably smaller than the cell diameter in order to avoid a spurious effect in the quality of transmitted polarizations. The light transmitted through the cell is collected into a photodetector, and a second identical photodetector is used to reduce the effect of the laser amplitude noise [15]. The differential signal is subsequently demodulated with a lock-in amplifier at different harmonics $q$ of $\Omega$. Three independent orthogonal coil systems supplied by high stability voltage-controlled-current sources, are used to generate the magnetic-field components [26]. The magnetic-field modulus $B$ is nominally $0.5 \mu \mathrm{T}$ corresponding to a Larmor frequency of $\omega_{L} \approx 2 \pi \times 2 \mathrm{kHz}$.

The experimental resonances are fitted with a Lorentzian or dispersive function multiplied by an amplitude parameter ( $A_{q}$ or $D_{q}$ ) or a combination of the two functions, as predicted by the model. Following the model prediction, we also use two different parameters for the linewidth of resonance at $\omega_{L}$ (and its subharmonics), and at $2 \omega_{L}$ (and its subharmonics), $\Gamma_{2}$ and $\Gamma_{1}$, respectively. In order to efficiently compare theoretical and experimental spectra they are normalized using a single scaling factor: the amplitude of the resonance demodulated in second harmonic recorded at $2 \omega_{L}$.

We then define the theoretical scaling factor by setting $q=2$ and $n=1$ in Eq. (17a), whereas the experimental scaling factor is obtained by fitting the $q=2, n=1$ resonance with a purely absorptive Lorentzian function. This simple normalization procedure allows us to get rid of the technical specifications of our experimental apparatus, and of the atomic system. One could remark that for $q=2$ and $n=1$ the amplitude of the expected [see Eqs. (17a) and (17b)] in-phase part of the lock-in signal is two times larger with respect to the quadrature part, as has been observed in the case of polarization modulation between orthogonal circularly polarized light states [16].

The theoretical linewidths are calculated using Eq. (11) where the population and coherence relaxation rates, i.e., $\gamma_{0}$ and $\gamma_{i}, i=1,2$, have been estimated by using the ground-state Hanle effect based on atomic alignment [27]. The method to estimate the relaxation rates is very similar to the one described in Ref. [28]. We have obtained $\gamma_{0} \approx 6 \mathrm{~Hz}, \gamma_{1} \approx 9 \mathrm{~Hz}$, and $\gamma_{2} \approx 11 \mathrm{~Hz}$. 
Notice that thanks to the amplitude normalization and the independent determination of the relaxation rates, we compare theory and experiments without adding free fit parameters.

\section{ANALYSIS OF THE FULL SPECTRUM AS A FUNCTION OF THE DETECTION HARMONICS}

In the following we discuss the full spectra and the resonance linewidths and amplitudes separately for the even and the odd demodulating harmonics.

Odd harmonics. In Fig. 2 the in-phase and quadrature part of the lock-in signal for the first and the third detection harmonics are shown. The black lines are the experimental spectra while the red-dashed lines are the model results. In perfect agreement with the model prediction, we observe that the $q$ th odd harmonics experimental spectrum $(\Omega>0)$ is composed only by two resonances occurring when $\Omega$ matches either $\omega_{L} / q$ or $2 \omega_{L} / q$.

From direct inspection of Eqs. (16) we found that for the opportune choice of lock-in phase, fixed $q$ for both the amplitude of the in-phase (absorptive) and quadrature (dispersive) signals, are equal, $A_{q}=D_{q}$ for resonance at $\omega_{L} / q$ $\left(2 \omega_{L} / q\right)$, while decreasing proportionally to $q^{-2}$.

We define the relative amplitude as the ratio between the quadrature and the in-phase amplitude either calculated or determined by fit of the experimental data, $D_{q} / A_{q}=1$. In Fig. 3 we report the experimental amplitude for the in-phase signal at $2 \omega_{L}$; the dashed line accounts for the $1 / q^{2}$ dependence of the resonance amplitudes predicted by Eq. (16a), and it is simply calculated by dividing the experimental amplitudes $A_{1}$ by $q^{2}$.

The same dependence on $q^{2}$ is predicted for all signal components both at $2 \omega_{L}$ and $\omega_{L}$. In Fig. 3 inset the experimental relative amplitudes $\left(D_{q} / A_{q}\right)$ of lock-in signals are plotted versus the detection harmonic $q$.

Resonances at $2 \omega_{L}$ and $\omega_{L}$ are expected to have slightly different linewidths. For $\theta=45^{\circ}$ one would expect $\Gamma_{1} / 2 \pi=$ $9.55 \mathrm{~Hz}$ and $\Gamma_{2} / 2 \pi=8.46 \mathrm{~Hz}$ from Eqs. (11). In Fig. 4 we plot the linewidth obtained from the fit of in-phase and quadrature resonances at $\omega_{L} / q$ and $2 \omega_{L} / q$. For fixed $q$ and $\omega_{L}$ the linewidth of the in-phase and quadrature signals are essentially equal within fluctuations of a few $\%$. We calculate from the values of experimental resonances $\Gamma_{1} / 2 \pi=8.2(2)$
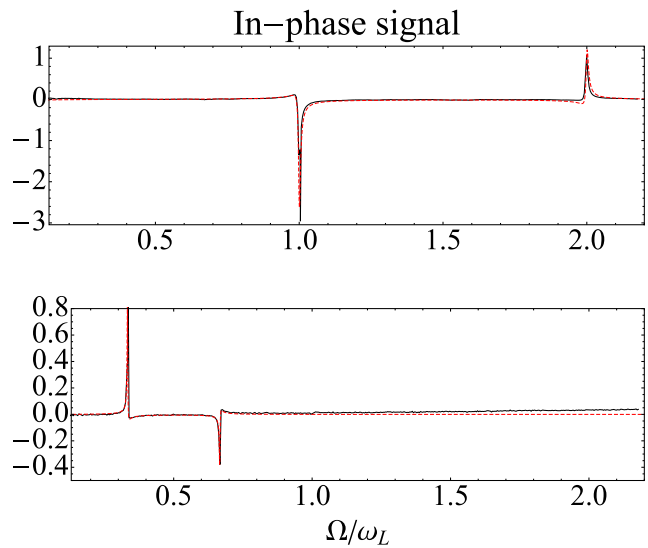

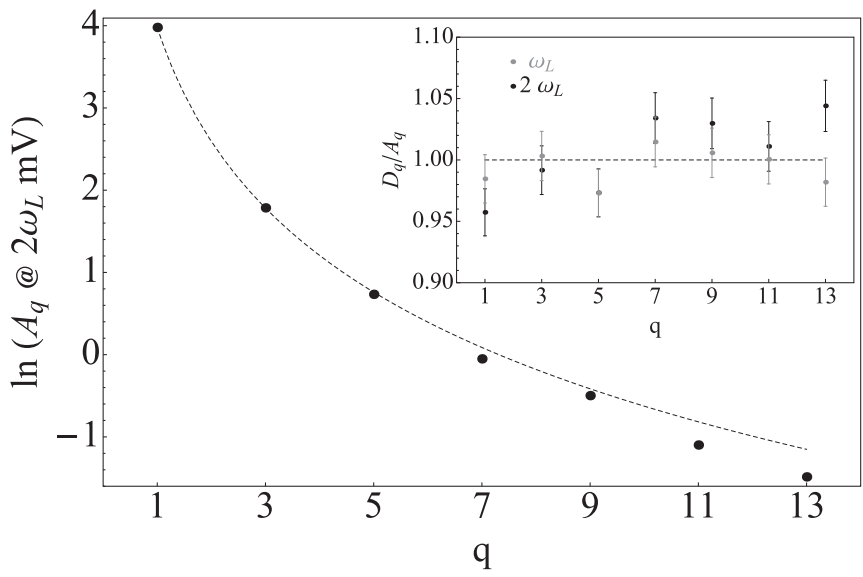

FIG. 3. Amplitude of the in-phase (absorptive) part of the lock-in resonance at $\Omega=2 \omega_{L}$ as a function of the detection harmonic for odd $q$ value, i.e., $q=1,3,5,7,9,11,13$. The dashed line is obtained dividing $A_{1}$ by $q^{2}$. Inset: Relative amplitude of the resonances at $\Omega=2 \omega_{L}$ (black) and $\omega_{L}$ (gray); the relative amplitude defined as $D_{q} / A_{q}$ for each resonance is expected to be 1 .

and $\Gamma_{2} / 2 \pi=8.1(9) \mathrm{Hz}$; those values differ $15 \%$ and $4 \%$ from theoretical predictions, respectively.

Even harmonics. In Fig. 5 the in-phase and quadrature lockin spectrum as a function of the modulation frequency are represented for $q=2$ and 4 . The spectrum is enriched, as explained above, by subharmonic resonances at $\omega_{L} / n$ and $2 \omega_{L} / n$, where $n$ is an odd integer.

One could remark that in Fig. 5 the outermost resonance at $\left(\Omega / \omega_{L}=2\right)$ is not perfectly symmetric. This effect is due to imperfections in the time response of the EOM to the $\Omega$ sweep, and can be completely suppressed by reducing the frequency range and by locally optimizing the lock-in phase.

We verified the behavior of resonance parameters (amplitude and linewidth) as a function of $q$ and $n$.

The relative amplitude, $D_{q} / A_{q}$ of the resonance at $\Omega=$ $2 \omega_{L} / n$ is proportional to $q / n$, as shown in Fig. 6 where it is plotted as a function of $q$ for different values of $n$. The dots represent experimental relative amplitudes while the lines mark the $(q / n)$ trend.
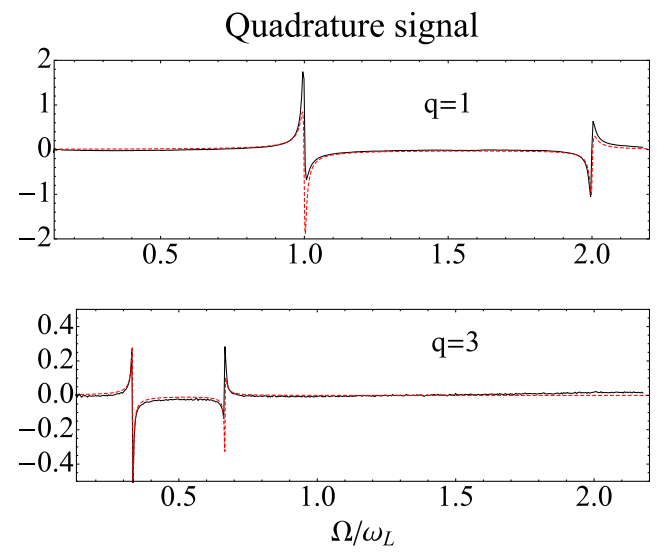

FIG. 2. (Color online) In-phase and quadrature lock-in signals at the first $(q=1)$ and third $(q=3)$ harmonic of $\Omega$ normalized taking into account the appropriate scaling factors: black and red-dashed lines represent experiment and model results, respectively. The magnetic-field direction is defined by $\theta=45^{\circ}$ and $\varphi=120^{\circ}$. 


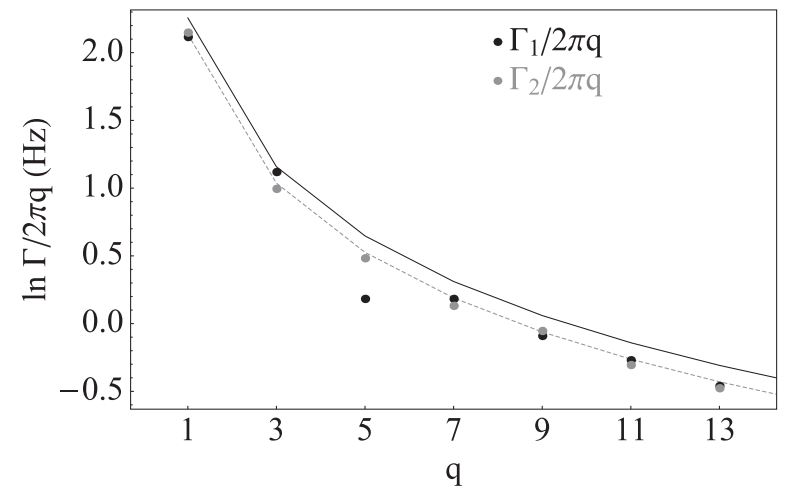

FIG. 4. Linewidth $(\Gamma / 2 \pi)$ of resonances at $\omega_{L} / q$ and $2 \omega_{L} / q$ as a function of $q$. The dots are the average values obtained by fit of the experimental resonances. The lines are calculated accounting for the $1 / q$ dependence of the $\Gamma_{i}$, where the initial values, i.e., $\Gamma_{1} / 2 \pi=$ $9.55 \mathrm{~Hz}$ and $\Gamma_{2} / 2 \pi=8.46 \mathrm{~Hz}$ at $q=1$, are calculated for the set of experimental parameters by applying Eqs. (11).

We studied the resonance subharmonics from $n=1$ up to $n=9$ for $2 \omega_{L}$ group of resonances. The same dependence is expected in the $\omega_{L}$ group of resonances. In contrast to what we observe for odd $-q$ detection, the linewidth of the resonances does not depend on $q$ but it does on $n$. In order to reduce the number of fitting parameters, the resonances at $2 \omega_{L} / n$ and $\omega_{L} / n$ are fitted with $\Gamma_{1} / n$ and $\Gamma_{2} / n$ linewidths.

In Fig. 7 the values of $\Gamma_{1}\left(\Gamma_{2}\right)$ from a multiresonance fit for a given $q$ are shown. The solid lines mark the average value of the data set, i.e., $\Gamma_{1} / 2 \pi=9.3(7) \mathrm{Hz}$ and $\Gamma_{2} / 2 \pi=8(1) \mathrm{Hz}$, while the dashed line is the theoretical value calculated from Eqs. (11) corresponding to $\Gamma_{1} / 2 \pi=9.55 \mathrm{~Hz}$ and $\Gamma_{2} / 2 \pi=$ $8.46 \mathrm{~Hz}$.

\section{DEPENDENCE OF THE $\omega_{L}$ AND $2 \omega_{L}$ MAGNETIC RESONANCES ON THE MAGNETIC-FIELD DIRECTION}

We fix $q=2$, and study the amplitude and linewidth of the resonance at $\omega_{L}$ and $2 \omega_{L}$ as a function of the magnetic field direction.
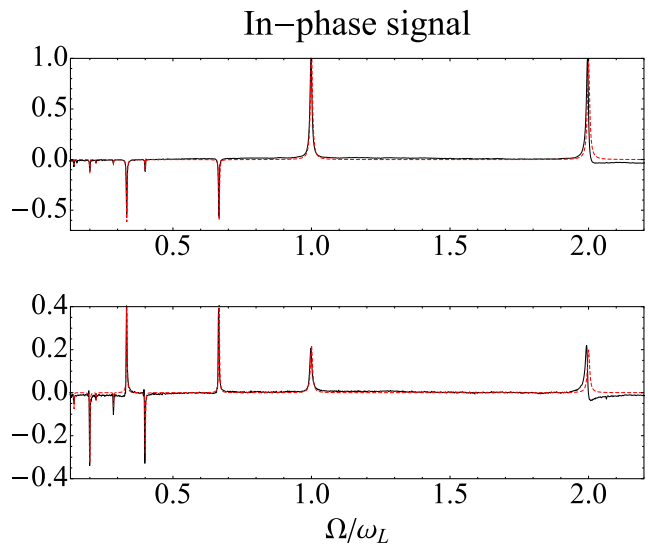

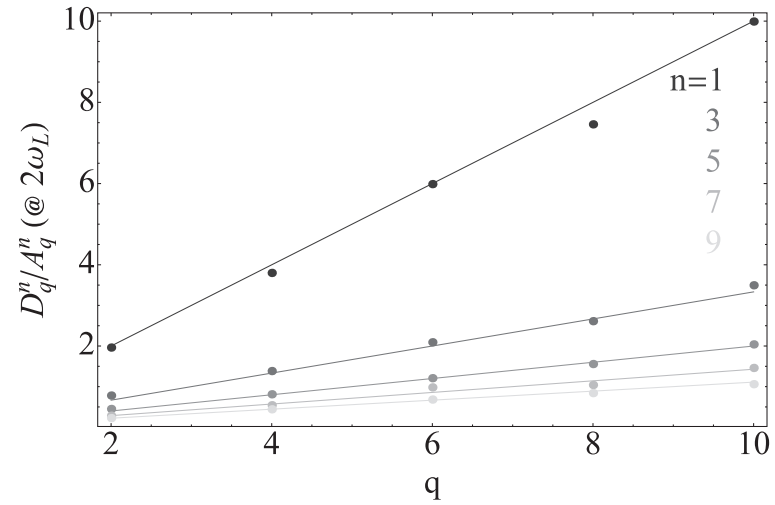

FIG. 6. Relative amplitude of the $2 \omega_{L}$ group of resonances as a function of the detection harmonic for even $q$ value, i.e., $q=$ $2,4,6,8,10$. We have studied the subharmonic resonances up to $n=9$.

The array of plots in Fig. 8 shows the amplitude of the experimental resonances at $2 \omega_{L}$ and $\omega_{L}$ which are expressed in terms of normalized amplitudes related to the $C_{1}$ and $C_{2}$ coefficients. The agreement between theory and experiment is excellent. We observe that the amplitudes oscillate with a period of $\pi / 2$ which is compatible with the alignment symmetry, when the resonance at $2 \omega_{L}$ amplitude reaches its maximum value, the resonance at $\omega_{L}$ amplitude is at its minimum, and vice versa.

It is worthwhile to remark that the minimum values of the resonance amplitudes do not reach exactly zero. In the worst case, i.e., for $\theta=90^{\circ}$, the contrast of resonances is noticeably reduced, however resonances are recordable during the experiments. This is not in contrast with the model predictions as commented in the Fig. 8 caption. The linewidths, $\Gamma_{1}$ and $\Gamma_{2}$, do not show any dependence on the azimuthal angle $\phi$, while they show a weak dependence on $\theta$ that is represented in Fig. 9. The experimental points in the figure are the average values of the linewidth obtained by fitting the 36 resonances (18 in-phase and 18 quadrature signals) recorded for different $\phi$. The linewidth variation on the $(0, \pi)$ range is about $20 \%$. $\Gamma_{1}$ and $\Gamma_{2}$ oscillate with a period $\pi$ and the phase difference is $\pi / 2$. The lines in Fig. 9 are calculated from Eqs. (11) by
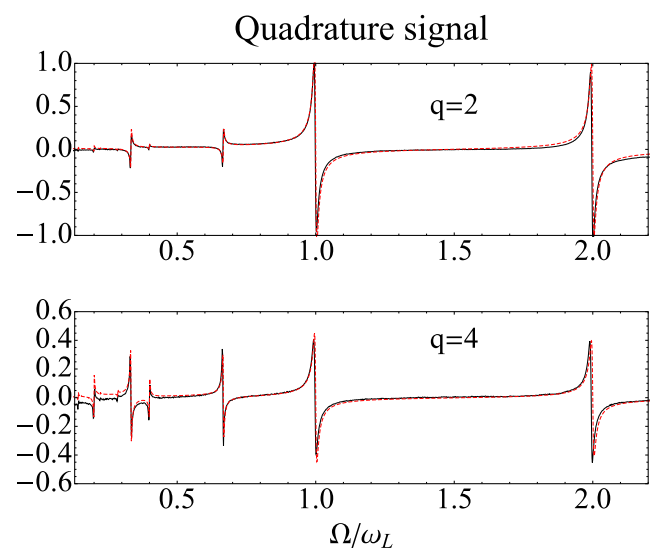

FIG. 5. (Color online) In-phase (absorptive) and quadrature (dispersive) lock-in signal at the second $(q=2)$ and fourth $(q=4)$ harmonic of the modulation frequency, as a function of the dimensionless ratio between the modulation frequency $\Omega$ and the Larmor frequency $\omega_{L}$. The spectra are normalized with the appropriate scaling factors. The angles $\theta$ and $\phi$ are $45^{\circ}$ and $50^{\circ}$. Black and dashed-red lines represent experiment and model results, respectively. 

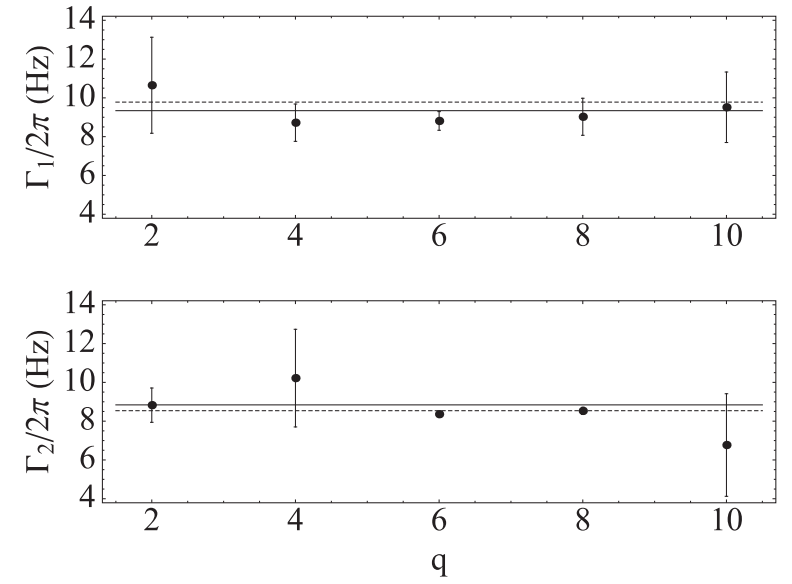

FIG. 7. The linewidths $\Gamma_{1}$ and $\Gamma_{2}$ of the resonance at $2 \omega_{L}$ (upper plot) and $\omega_{L}$ (lower plot): dots are obtained by fitting the experimental resonances, solid lines are the average experimental values, i.e., 9.3(7) $\mathrm{Hz}$ and $8(1) \mathrm{Hz}$, while the dotted lines are the theoretical predictions for $\theta=45^{\circ}$, i.e. 9.55 and $8.46 \mathrm{~Hz}$, respectively.
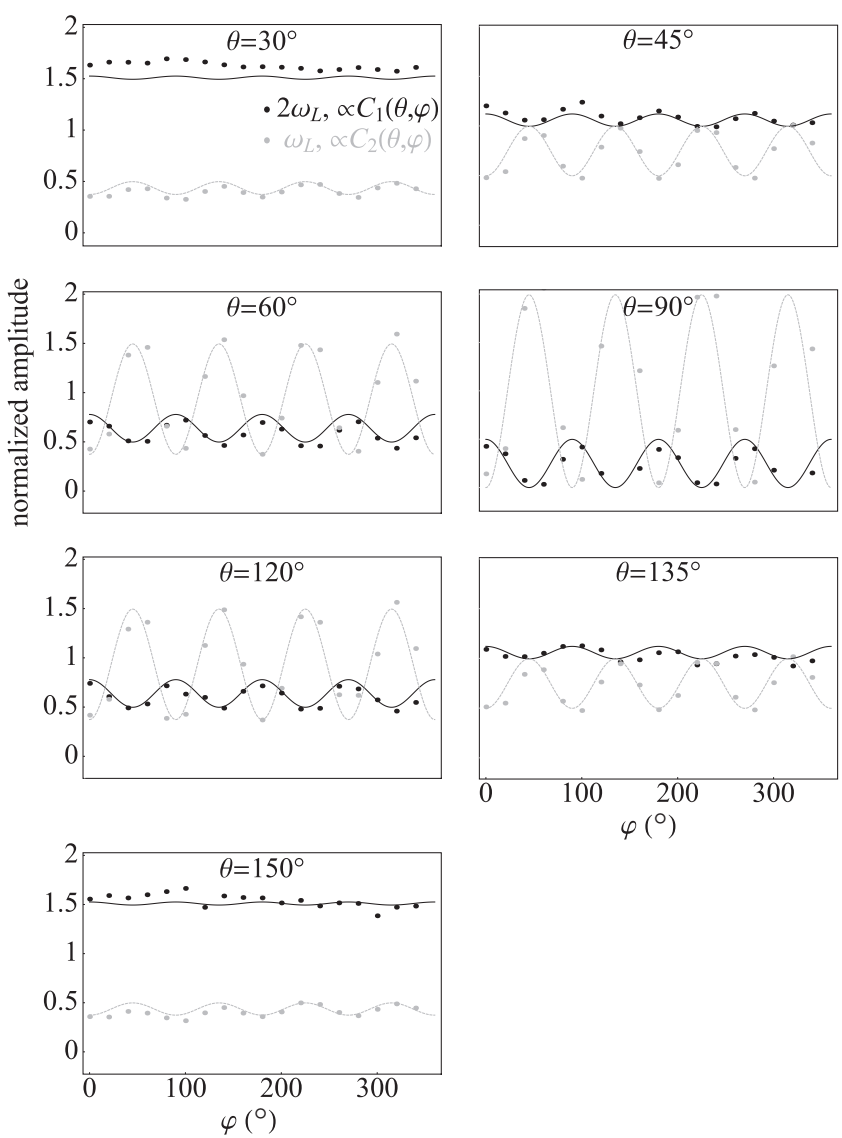

FIG. 8. Experimental (dots) and theoretical (line) values for the normalized amplitudes $\left(\propto C_{1}\right.$ and $\left.C_{2}\right)$ of $2 \omega_{L}$ and $\omega_{L}$ resonance alternate in a complementary way when the direction of the magnetic field is varied. We remark that in the $\theta=90^{\circ}$ inset, the experimental amplitude minima do not reach exactly zero, even though strongly suppressed; the resonances are clearly visible. This is not in contrast with the model predictions when all orders in $\eta$ are taken into account, as we have verified by numerical simulation.

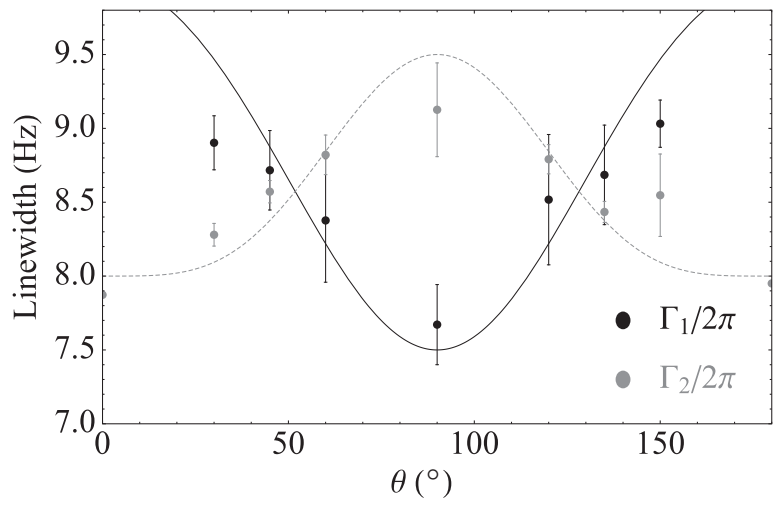

FIG. 9. Linewidth of resonance at $\omega_{L}\left(\Gamma_{2} / 2 \pi\right)$, and $2 \omega_{L}\left(\Gamma_{1} / 2 \pi\right)$ as a function of $\theta$ : dots are experiments and line is Eqs. (11).

using $\gamma_{0}=6 \mathrm{~Hz}, \gamma_{1}=8 \mathrm{~Hz}$, and two slight different values for $\gamma_{2}=10 \mathrm{~Hz}\left(\Gamma_{2}\right)$ and $\gamma_{2}=11\left(\Gamma_{1}\right)$. In the worst case the values used for relaxation rates are in $10-15 \%$ agreement with the measured ones.

The oscillatory complementary behavior of both amplitude and linewidth of the two groups of resonances excited in the proposed configuration can be interesting in view of a coupled atomic magnetometer similar to the one developed in Graz [29]. A feedback loop magnetometer uses an error signal to monitor the frequency fluctuations proportional to the magnetic-field fluctuations. The error signal can be the dispersive part or the phase of the lock-in signal. In both cases the magnetometer sensitivity is proportional to the ratio between the linewidth and the amplitude of the resonance. However we want to underline that the resonances at $\omega_{L}$ and $2 \omega_{L}$ are never simultaneously suppressed. The information carried by the two resonances can be exploited to build up a dead-zone free magnetometer with reduced sensitivity losses. In addition, after appropriate characterization of the magnetometer, it could be interesting to study the possibility of exploiting these structured spectra to realize a vectorial magnetometer.

All experiments are carried out at low laser power, i.e., in the range of validity of the theory, far from optimal operating magnetometer conditions. However we expect such an interaction scheme will reach the same performance of the other magnetometers based on similar approaches, i.e., pT sensitivity down to tens of fT sensitivity in the shot-noise limit expectation.

\section{CONCLUSIONS}

We studied the magneto-optical resonances prepared with a laser whose polarization alternates between two linear orthogonal states focusing our attention to the $F_{g}=3 \rightarrow F_{e}=4$ transitions which is a good candidate for high performance atomic magnetometer. This interaction scheme is characterized by the synchronous excitation of atomic alignment, which is the base of the push-pull optical pumping technique. A model is developed taking into account the low laser power and the separation of the time scales. We characterize the resonance spectra as a function of the lock-in detection harmonic observing two independent resonance groups at the Larmor frequency and its second harmonic. We study the dependence of amplitude and linewidth of the 
two groups of resonances for 126 magnetic-field directions observing that they alternate. Utilizing both resonances at $\omega_{L}$ and $2 \omega_{L}$ a dead-zone free magnetometer can be built in which sensitivity losses depending on the magnetic-field direction can be reduced. Finally the symmetric synchronous pumping of atomic alignment in principle reduces also the so-called heading errors as discussed in [19].

\section{ACKNOWLEDGMENTS}

The authors thank Y. Dancheva and V. Biancalana for carefully reading the manuscript. E.B. thanks the SNF-Ambizione, Grant No. PZ00P2_131926, for financial support; her project was hosted and technically supported by Professor Weis, head of the Fribourg Atomic Physics group, and his collaborators, in particular Z. D. Grujić for his technical advice.

\section{APPENDIX: DERIVATION OF THE PUMPING RATES}

In this Appendix we derive, for the sake of completeness, the pumping rate terms $S_{q}^{k}$ appearing in Eq. (5). We follow mainly the method outlined in the paper of Dumont [30]. The density-matrix description of an ensemble of degenerate twolevel atoms can be written as

$$
\rho=\left(\begin{array}{ll}
e e \rho & e g \rho \\
g e & \text { gg } \rho
\end{array}\right)
$$

where $e_{e} \rho$ and ${ }_{g g} \rho$ are Hermitian square matrices of dimension $\left(2 F_{e}+1\right) \times\left(2 F_{e}+1\right)$ and $\left(2 F_{g}+1\right) \times\left(2 F_{g}+1\right)$ describing the excited and ground state respectively, while the optical coherence ${ }_{e g} \rho\left(=_{g e} \rho^{\dagger}\right)$ is a rectangular matrix of dimension $\left(2 F_{e}+1\right) \times\left(2 F_{g}+1\right)$. We use the multipole moments representation of the density matrix [21,22,30,31] operator because their transparent physical meaning allows for noticeable description of magneto-optical resonances (see for instance the discussion in [20] and references therein). Then, $\rho$ reads

$$
\rho=\sum_{i, j=g, e} \sum_{k, q}{ }_{i j} \rho_{q}^{k}{ }_{i j} T_{q}^{k},
$$

where the coefficients ${ }_{i j} \rho_{q}^{k}$ are the multipole moments and the operators ${ }_{i j} T_{q}^{k}$, using the $3 J$ symbol, take the usual form $[21,22]$

$$
\begin{aligned}
{ }_{i j} T_{q}^{k} \equiv & \sqrt{2 k+1} \sum_{M}(-1)^{F_{i}+M}\left(\begin{array}{ccc}
F_{i} & F_{j} & k \\
M & q-M & -q
\end{array}\right) \\
& \times\left|F_{i}, M\right\rangle\left\langle F_{j}, M-q\right| .
\end{aligned}
$$

Notice that a complete description of the problem requires the multipole moments related to both ground $\left({ }_{g g} \rho_{q}^{k}\right)$ and excited $\left(e e \rho_{q}^{k}\right)$ Zeeman multiplets as well as those related to the optical coherences $\left({ }_{e g} \rho_{q}^{k}\right.$ and $\left.{ }_{g e} \rho_{q}^{k}\right)$. A common and simplified notation used in the literature [20] for the groundstate multipole moments is ${ }_{g g} \rho_{q}^{k} \equiv m_{q}^{k}$ as used in the main text.

In the presence of a laser field resonant with the $F_{g} \rightarrow F_{e}$ transition, the atom-laser interaction Hamiltonian $H_{A L}=-\mathbf{d}$. $\mathbf{E}$, where $\mathbf{d}$ and $\mathbf{E}=E_{0}\left[\boldsymbol{\epsilon} \mathrm{e}^{-i \omega t}+\boldsymbol{\epsilon}^{*} \mathrm{e}^{+i \omega t}\right]$ are the electric dipole and the laser electric field, respectively, can be written in the rotating wave approximation (RWA) frame as [31]

$$
\begin{aligned}
H_{A L}= & -\frac{\left\langle F_{e}\|d\| F_{g}\right\rangle E_{0}}{\sqrt{3}} \sum_{q=-1}^{1}(-1)^{q}\left[\epsilon_{-q e g} T_{q}^{1}\right. \\
& \left.+(-1)^{F_{g}+F_{e}}\left(\epsilon^{*}\right)_{-q g e} T_{q}^{1}\right] .
\end{aligned}
$$

The dynamic evolution of the atomic ensemble is given by the solution of the optical Bloch equations. Using standard methods and the form (A4) of the interaction, the optical Bloch equations in the RWA frame become

$$
\begin{aligned}
& { }_{g g} \dot{\rho}_{q}^{k}=\Theta(k)_{e e} \rho_{q}^{k}-i \Omega_{R} \sum_{k^{\prime}, q^{\prime}}\left[A_{k^{\prime}, q^{\prime}}^{k, q} \rho_{q^{\prime}}^{k^{\prime}}+B_{k^{\prime}, q^{\prime}}^{k, q} \rho_{q^{\prime}}^{k^{\prime}}\right], \\
& e e \dot{\rho}_{q}^{k}=-\Gamma_{e e} \rho_{q}^{k}+i \Omega_{R} \sum_{k^{\prime}, q^{\prime}}\left[C_{k^{\prime}, q^{\prime}}^{k, q} \rho^{\prime} \rho_{q^{\prime}}^{k^{\prime}}+D_{k^{\prime}, q^{\prime}}^{k, q} \rho_{q^{\prime}}^{k^{\prime}}\right], \\
& { }_{g e} \dot{\rho}_{q}^{k}=\left(-\frac{\Gamma}{2}+i \delta\right) g_{g e} \rho_{q}^{k} \\
& +i \Omega_{R} \sum_{k^{\prime}, q^{\prime}}\left[F_{k^{\prime}, q^{\prime}}^{k, q} \text { ee } \rho_{q^{\prime}}^{k^{\prime}}+G_{k^{\prime}, q^{\prime}}^{k, q g} \rho_{q^{\prime}}^{k^{\prime}}\right], \\
& { }_{e g} \dot{\rho}_{q}^{k}=\left(-\frac{\Gamma}{2}-i \delta\right){ }_{e g} \rho_{q}^{k} \\
& +i \Omega_{R} \sum_{k^{\prime}, q^{\prime}}\left[H_{k^{\prime}, q^{\prime}}^{k, q} \text { ee } \rho_{q^{\prime}}^{k^{\prime}}+L_{k^{\prime}, q^{\prime} g g}^{k, q} \rho_{q^{\prime}}^{k^{\prime}}\right],
\end{aligned}
$$

which have the same structure as in the nondegenerate twolevel system. Here $\Gamma$ is the rate of spontaneous emission $F_{e} \rightarrow F_{g}, \delta \equiv \omega-\omega_{e g}$ is the optical detuning, and $\Omega_{R}=$ $\left\langle F_{e}\|d\| F_{g}\right\rangle E_{0} / \sqrt{3}$ is the laser Rabi frequency (we assume $\hbar \equiv 1$ through the paper). The coefficients

$$
\Theta(k) \equiv(-1)^{1+k+F_{g}+F_{e}}\left(2 F_{e}+1\right)\left\{\begin{array}{lll}
F_{e} & F_{e} & k \\
F_{g} & F_{g} & 1
\end{array}\right\} \Gamma
$$

describe the feeding of the ground-state quantities due to spontaneous emission [30]. The explicit expression for the coefficients like $A_{k^{\prime}, q^{\prime}}^{k, q}$ are given in terms of scalar products,

$$
\begin{aligned}
A_{k^{\prime}, q^{\prime}}^{k, q} & =\boldsymbol{\epsilon} \cdot\left({ }_{g g e} \boldsymbol{\Lambda}_{k^{\prime}, q^{\prime}}^{k, q}\right), \\
B_{k^{\prime}, q^{\prime}}^{k, q} & =(-1)^{F_{g}+F_{e}+k^{\prime}+k} \boldsymbol{\epsilon}^{*} \cdot\left({ }_{g g e} \boldsymbol{\Lambda}_{k^{\prime}, q^{\prime}}^{k, q}\right), \\
C_{k^{\prime}, q^{\prime}}^{k, q} & =(-1)^{1+k^{\prime}+k} \boldsymbol{\epsilon} \cdot\left({ }_{e e g} \boldsymbol{\Lambda}_{k^{\prime}, q^{\prime}}^{k, q}\right), \\
D_{k^{\prime}, q^{\prime}}^{k, q} & =(-1)^{F_{g}+F_{e}+1} \boldsymbol{\epsilon}^{*} \cdot\left({ }_{e e g} \boldsymbol{\Lambda}_{k^{\prime}, q^{\prime}}^{k, q}\right), \\
F_{k^{\prime}, q^{\prime}}^{k, q} & =(-1)^{F_{g}+F_{e}+k^{\prime}+k+1} \boldsymbol{\epsilon}^{*} \cdot\left(_{e g e} \boldsymbol{\Lambda}_{k^{\prime}, q^{\prime}}^{k, q}\right), \\
G_{k^{\prime}, q^{\prime}}^{k, q} & =(-1)^{F_{g}+F_{e}+1} \boldsymbol{\epsilon}^{*} \cdot\left({ }_{g e g} \boldsymbol{\Lambda}_{k^{\prime}, q^{\prime}}^{k, q}\right), \\
H_{k^{\prime}, q^{\prime}}^{k, q} & =-\boldsymbol{\epsilon} \cdot\left({ }_{e g e} \boldsymbol{\Lambda}_{k^{\prime}, q^{\prime}}^{k, q}\right), \\
L_{k^{\prime}, q^{\prime}}^{k, q} & =(-1)^{k^{\prime}+k+1} \boldsymbol{\epsilon} \cdot\left({ }_{g e g} \boldsymbol{\Lambda}_{k^{\prime}, q^{\prime}}^{k, q}\right),
\end{aligned}
$$


where the $\sigma$ spherical component of the $\boldsymbol{\Lambda}$ vector, that couples different multipoles, is given by

$$
\begin{aligned}
\left({ }_{a b c} \Lambda_{k^{\prime}, q^{\prime}}^{k, q}\right)_{\sigma}= & (-1)^{F_{a}+F_{b}-q} \sqrt{3(2 k+1)\left(2 k^{\prime}+1\right)} \\
& \times\left(\begin{array}{ccc}
1 & k^{\prime} & k \\
\sigma & q^{\prime} & -q
\end{array}\right)\left\{\begin{array}{ccc}
1 & k^{\prime} & k \\
F_{a} & F_{b} & F_{c}
\end{array}\right\} .
\end{aligned}
$$

The solution of the (A5) can be pursued only by numerical methods for intermediate and high laser power.

However, if the laser intensity is weak so that the transition is not saturated, $\Omega_{R} / \Gamma \ll 1$ and $\Omega_{R}$ can used as a perturbative parameter, that is assuming

$$
{ }_{i j} \rho_{q}^{k}=\sum_{n=0}^{\infty} \Omega_{R}^{n}{ }_{i j}^{n} \rho_{q}^{k},
$$

Eqs. (A5) can be solved easily. It is well known [30] that the optical coherence multipoles get only odd order contributions while the ground- and excited-state multipoles have only the even ones. Assuming an initial (before laser action) thermodynamical equilibrium ${ }_{i j}^{0} \rho_{q}^{k}=\sqrt{2 F_{g}+1} \delta_{i, g} \delta_{j, g} \delta_{k, 0} \delta_{q, 0}$, one obtains the first-order quantities

$$
\begin{aligned}
& { }_{g e}^{1} \rho_{q}^{k}=i\left(2 F_{g}+1\right)^{-1 / 2} \frac{1}{\Gamma / 2-i \delta} G_{0,0}^{k, q}, \\
& { }_{e g}^{1} \rho_{q}^{k}=i\left(2 F_{g}+1\right)^{-1 / 2} \frac{1}{\Gamma / 2+i \delta} L_{0,0}^{k, q},
\end{aligned}
$$

where $G_{0,0}^{k, q}$ and $L_{0,0}^{k, q}$ are easily computed as

$$
G_{0,0}^{k, q}=(-1)^{F_{g}+F_{e}+1+q}\left(2 F_{g}+1\right)^{-1 / 2}\left(\epsilon^{*}\right)_{-q} \delta_{k, 1},
$$

$$
L_{0,0}^{k, q}=(-1)^{k+1+q}\left(2 F_{g}+1\right)^{-1 / 2}(\epsilon)_{-q} \delta_{k, 1} .
$$

To compute second-order quantities one needs sums like $\sum_{k^{\prime}, q^{\prime}} A_{k^{\prime}, q^{\prime}}^{k, q} G_{0,0}^{k^{\prime}, q^{\prime}}$ which can be expressed after some algebra introducing the laser field multipoles (2). For instance

$$
\begin{aligned}
\sum_{k^{\prime}, q^{\prime}} A_{k^{\prime}, q^{\prime}}^{k, q} G_{0,0}^{k^{\prime}, q^{\prime}}= & 3(-1)^{F_{g}+F_{e}+1+q}\left(2 F_{g}+1\right)^{-1 / 2} \\
& \times\left\{\begin{array}{ccc}
1 & 1 & k \\
F_{g} & F_{g} & F_{e}
\end{array}\right\} \mathbb{E}_{-q}^{k} .
\end{aligned}
$$

We do not explicitly report the other sums which can be elaborated along the same steps of this one. After some algebra the second-order ground-state multipole pumping rates $S_{q}^{k}$ are found to be

$$
S_{q}^{k}=3 \Omega_{R} \frac{\Gamma}{(\Gamma / 2)^{2}+\delta^{2}} \mathcal{R}_{k}(-1)^{q} \mathbb{E}_{-q}^{k},
$$

where the $\mathcal{R}_{k}$ coefficients are

$$
\begin{aligned}
\mathcal{R}_{k}= & \frac{1}{2 F_{g}+1}\left[(-1)^{F_{g}+F_{e}+1}\left\{\begin{array}{ccc}
1 & 1 & k \\
F_{g} & F_{g} & F_{e}
\end{array}\right\}\right. \\
& \left.-\left(2 F_{e}+1\right)\left\{\begin{array}{ccc}
1 & 1 & k \\
F_{e} & F_{e} & F_{g}
\end{array}\right\}\left\{\begin{array}{lll}
F_{e} & F_{e} & k \\
F_{g} & F_{g} & 1
\end{array}\right\}\right] .
\end{aligned}
$$

For the transition $F_{g}=4 \rightarrow F_{e}=3$ the only $\mathcal{R}_{k}$ different from zero are $\mathcal{R}_{1}=-\sqrt{30} / 1296 \approx-4 \times 10^{-3}$ and $\mathcal{R}_{2}=$ $\sqrt{462} / 3888 \approx 5 \times 10^{-3}$.
[1] E. B. Alexandrov, M. Auzinsh, D. Budker, D. F. Kimball, S. M. Rochester, and V. V. Yashchuk, J. Opt. Soc. Am. B 22, 7 (2005).

[2] A. L. Bloom, Appl. Opt. 1, 61 (1962).

[3] W. E. Bell and A. L. Bloom, Phys. Rev. Lett. 6, 280 (1961).

[4] J. Belfi, G. Bevilacqua, V. Biancalana, Y. Dancheva, and L. Moi, J. Opt. Soc. Am. B 24, 1482 (2007).

[5] V. Schultze, R. I. Jsselsteijn, T. Scholtes, S. Woetzel, and H.-G. Meyer, Opt. Express 20, 14201 (2012).

[6] W. Gawlik, L. Krzemień, S. Pustelny, D. Sangla, J. Zachorowski, M. Graf, A. O. Sushkov, and D. Budker, Appl. Phys. Lett. 88, 131108 (2006).

[7] D. Budker, D. F. Kimball, V. V. Yashchuk, and M. Zolotorev, Phys. Rev. A 65, 055403 (2002).

[8] D. Budker and M. Romalis, Nat. Phys. 3, 227 (2007).

[9] G. Bevilacqua, V. Biancalana, Y. Dancheva, and L. Moi, Phys. Rev. A 85, 042510 (2012).

[10] S. Xu, M. H. Donaldson, A. Pines, S. M. Rochester, D. Budker, and V. V. Yashchuk, Appl. Phys. Lett. 89, 224105 (2006).

[11] G. Bevilacqua, V. Biancalana, Y. Dancheva, and L. Moi, Annual Reports on NMR Spectroscopy 78, 103 (2013).

[12] T. Theis, P. Ganssle, G. Kervern, S. Knappe, J. Kitching, M. P. Ledbetter, D. Budker, and A. Pines, Nat. Phys. 7, 571 (2011).
[13] A. Ben-Kish and M. V. Romalis, Phys. Rev. Lett. 105, 193601 (2010).

[14] Z. D. Grujić and A. Weis, Phys. Rev. A 88, 012508 (2013).

[15] I. Fescenko, P. Knowles, A. Weis, and E. Breschi, Opt. Express 21, 15121 (2013).

[16] E. Breschi, Z. D. Gruijć, P. Knowles, and A. Weis, Phys. Rev. A 88, 022506 (2013).

[17] E. Breschi, Z. D. Grujić, P. Knowles, and A. Weis, Appl. Phys. Lett. 104, 023501 (2014).

[18] Y.-Y. Jau, E. Miron, A. B. Post, N. N. Kuzma, and W. Happer, Phys. Rev. Lett. 93, 160802 (2004).

[19] A. Weis, G. Bison, and A. S. Pazgalev, Phys. Rev. A 74, 033401 (2006).

[20] G. Bevilacqua, E. Breschi, and A. Weis, Phys. Rev. A 89, 033406 (2014).

[21] A. Omont, Prog. Quantum Electron. 5, 69 (1977).

[22] W. Happer, Rev. Mod. Phys. 44, 169 (1972).

[23] J. J. Sakurai and J. J. Napolitano, Modern Quantum Mechanics, 2nd ed. (Addison-Wesley, San Francisco, 2010).

[24] Z. D. Grujić, M. Mijailović, D. Arsenović, A. Kovačević, M. Nikolić, and B. M. Jelenković, Phys. Rev. A 78, 063816 (2008).

[25] N. Castagna, G. Bison, G. Di Domenico, A. Hofer, P. Knowles, C. Macchione, H. Saudan, and A. Weis, Appl. Phys. B 96, 763 (2009). 
[26] E. Breschi, Z. Gruijć, and A. Weis, Appl. Phys. B 115, 85 (2014).

[27] E. Breschi and A. Weis, Phys. Rev. A 86, 053427 (2012).

[28] N. Castagna and A. Weis, Phys. Rev. A 84, 053421 (2011); 85, 059907 (2012).
[29] A. Pollinger, M. Ellmeier, W. Magnes, C. Hagen, W. Baumjohann, E. Leitgeb, and R. Lammegger, IEEE Conf. Pub. 33 (2012).

[30] M. Dumont and B. Decomps, J. Phys. France 29, 181 (1968).

[31] C. Feuillade and P. R. Berman, Phys. Rev. A 29, 1236 (1984). 